\title{
Emanuele Navarro delle Miraglia, Macchiette parigine
}

\section{Annalisa Bottacin}

\section{(2) OpenEdition}

1 Journals

\section{Edizione digitale}

URL: https://journals.openedition.org/studifrancesi/45955

DOI: 10.4000/studifrancesi.45955

ISSN: 2421-5856

\section{Editore}

Rosenberg \& Sellier

\section{Edizione cartacea}

Data di pubblicazione: 1 octobre 2007

Paginazione: 454-455

ISSN: 0039-2944

\section{Notizia bibliografica digitale}

Annalisa Bottacin, «Emanuele Navarro delle Miraglia, Macchiette parigine», Studi Francesi [Online], 152 (LI | II) | 2007, online dal 30 novembre 2015, consultato il 24 novembre 2021. URL: http:// journals.openedition.org/studifrancesi/45955 ; DOI: https://doi.org/10.4000/studifrancesi.45955

Questo documento è stato generato automaticamente il 24 novembre 2021.

\section{(c) $(7)$}

Studi Francesi è distribuita con Licenza Creative Commons Attribuzione - Non commerciale - Non opere derivate 4.0 Internazionale. 


\title{
Emanuele Navarro delle Miraglia, Macchiette parigine
}

\author{
Annalisa Bottacin
}

\section{NOTIZIA}

EMANUELE NAVARRO DELLE MIRAGLIA, Macchiette parigine, a cura e con un saggio introduttivo di Pietro MILone, Milano, La Vita Felice, 2006 (Collana "Porte Aperte", n¹1. Le Letture di Leonardo Sciascia $\mathrm{n}^{\circ} 4$. Collana pubblicata in collaborazione con l'Associazione degli Amici di Leonardo Sciascia), pp. 233, ill.

1 In quell'alternanza che sovente verte intorno alle figure della letteratura destinatarie di un maggiore o minore ascolto da parte del pubblico, s'inserisce di netto quella, recentemente riscoperta e riproposta da Leonardo Sciascia, di Emanuele Navarro Della Miraglia, giornalista, scrittore, importante testimone di fondamentali avvenimenti storici e culturali, quali la partecipazione all'impresa garibaldina e la collaborazione ad alcune testate, tra cui l'«Indipendente» di Alexandre Dumas. Nato nella sicula Sambuca Zabut nel 1838, Navarro si stabilisce a Parigi negli anni Sessanta, dove segue, da inviato, gli avvenimenti della guerra franco-prussiana e della Comune. Rientrato in Italia nel 1872, si spegnerà a Roma nel 1919. Nel 1881, edita un volumetto, dal curioso titolo, Macchiette parigine, originali ritratti di alcune importanti figure dell'Ottocento francese. Come rileva Pietro Milone nel corposo saggio introduttivo dal titolo, Gran teatro del mondo: un siciliano a Parigi (pp. 9-68), «le Macchiette parigine, opera dalla scrittura piena di brio, di gusto e d'intelligenza, restano nella nostra storia letteraria, al pari (e anzi di più) delle ormai abbastanza note sue opere di narrativa di ambiente siciliano; e meritano di essere ricordate e analizzate in un ambito che si colloca tra giornalismo, letteratura e critica» (p. 13). L'operazione che Navarro riesce a effettuare con la sua scrittura, dal taglio tecnico perfetto, è quella di creare un allestimento scenico in cui situare, nello spazio rappresentativo, le figure più in vista del milieu politico-letterario di una Parigi di cui è spettatore attento e mordace, dove non mancano le occasioni di incontro. Ma non è altresì da tralasciare lo studio introspettivo del personaggio da 
tempo fuori scena, in quanto scomparso, come nel caso di Stendhal che apre la narrazione, mancato nel 1842, come ben evidenzia Leonardo Sciascia. In effetti la prima macchietta è infatti dedicata a Stendhal (pp. 73-78) che Navarro non conobbe e di cui riporta quanto poteva aver ascoltato nei salotti parigini e tratto dalla lettura della sua opera. Come lesse Luigi Foscolo Benedetto, che segnalò nel suo Arrigo Beyle Milanese l'edizione delle Macchiette del 1881, edita a Milano da Brignola, il suo è «in sé un profilo briosissimo, intelligente, sentito... ma anche talvolta banale e inconsistente» (L.F. Benedetto, Arrigo Beyle Milanese, Firenze, Sansoni, 1942, p. 100). E se, a nostro avviso, si può accusarlo di percorsi déjà vu, quali l'accenno a «una bella signora di Milano [che] lo chiamava scherzando: il Cinese» (la Pietragrua), certo non è da tralasciare il suo giudizio su «La Chartreuse de Parme [che s]arebbe un capolavoro se, in essa, lo stile fosse adeguato al concetto, se la mancanza di metodo non generasse una certa confusione, se i particolari inutili non frastagliassero troppo il racconto...» (pp. 76-77). Non vi è certo dell'amore per il grenoblese, ed è ciò che ribadisce anche Sciascia; pur tuttavia nel breve scritto, «ci facciamo l'idea che Navarro conosceva benissimo l'opera di Stendhal e che, malgrado tutto quel che di inamabile dice di trovarvi, l'amasse» (L. Sciascia, L'Adorabile Stendhal, Milano, Adelphi, 2003, p. 90). Il secondo di questi profili che, come riporta l'autore, «furono scritti, a intervalli, secondo il capriccio e l'occasione» (p. 71) relativi a scrittori, uomini politici, artisti, è dedicato ad Adolphe Thiers (pp. 79-84). «Non so che nome gli si darebbe in Italia - esordisce Navarro - a Parigi molti chiamano un parvenu» e ne narra l'avanzata sociale, condotta passo passo, quasi come fosse una disciplina da seguire; venuto a Parigi dalla provincia con l'amico Mignet, Thiers si dà subito un gran daffare per esporsi in società e frequentare i luoghi più in vista: «aveva dell'ingegno; aveva l'ardire, la petulanza, l'attività, la fiducia nelle proprie forze questa condizione madre del successo - e riuscì», (p. 80). La figura di Victor Hugo è esaltata, nell'omonimo ritratto (pp. 85-89): «è barone, è conte, è visconte? - si chiede Navarro - Non si sa bene. Forse non è nulla di tutto ciò. Ha un titolo migliore; è un uomo di genio...» (p. 85). A Parigi lo scrittore siciliano incontrò e si legò d'amicizia con una matura George Sand di cui traccia un ritratto (pp. 97-102) degno di una sognatrice romantica; ne esalta, pur brevemente, l'esistenza sin dagli anni giovanili, il suo matrimonio con il barone Dudevant, con cui «visse in campagna, leggendo molto, cucendo poco, suonando pianoforte, allattando i bambini comprimendo le aspirazioni vaghe che le tumultuavano confusamente nel cuore» (p. 97). Navarro analizza la sua figura di donna-scrittrice nella Parigi dell'epoca, con particolare riguardo ai suoi atteggiamenti che ben l'avanzarono sul suo tempo e che elogia: «nel nostro secolo, la signora Sand ha provato che si può essere donna e avere del genio; essa ha mostrato inoltre, coll'esempio, che l'emancipazione del sesso debole non deve e non può varcare certi limiti» (p. 101). Segue il medaglione dedicato a un grande amore di George Sand, Alfred de Musset (pp. 103-108), di cui Navarro ammira l'arte ma anche la bellezza: «Il poeta era biondo, delicato, bello, aveva la taglia fina e pieghevole, come una donna; sapeva annodare con arte la cravatta; amava i tacchi appuntati e alti; portava i capelli raccolti in un grosso ciuffo da un lato, e il cappello a cilindro, inchinato sull'orecchio, dall'altro» (p. 104). Navarro insiste a lungo, nelle pagine relative a Gautier (pp. 115-119) sul percorso artistico dell'autore di Mademoiselle de Maupin: «Siccome aveva il sentimento innato de' colori e delle linee, appena uscito dal collegio, studiò pittura. Però, la mano rispondeva male al pensiero, e le difficoltà dell'esecuzione lo scoraggiarono» (p. 115). Ecco che la scrittura diventa così cammino preferenziale $\mathrm{e}$ punto di convergenza di aspirazioni pittoriche, il che fa dire a Navarro che Gautier 
«scriveva come forse Benvenuto Cellini cesellava un gioiello, coprendolo di maravigliosi rabeschi, ma senza cercarne l'utilità pratica» (p. 116), evidenziando così in Gautier l'originale e completa teoria estetica, rivolta ad affermare l'autonomia e la libertà dell'arte, frutto di libera scelta dell'artista e svincolata da qualsiasi legame. Anche negli altri, vivaci trattarelli su questo o quel personaggio (Girardin, Proudhon, Barbey d'Aurevilly, Grévy, Baudelaire, Flaubert, Courbet, Dumas figlio, Rochefort, Sardou, Gambetta, Carpeaux, Daudet, Sarah Bernhardt), Navarro, come scrive Sciascia, «passa sempre in punta di penna, alla biografia, al carattere, al comportamento, alle opere. Ogni tanto suo malgrado c'è qualche lampo di simpatia e affiora il giudizio esatto e penetrante». (L. Sciascia, ivi, p. 105). 